\title{
COMMENTARY
}

\section{Pediatric facial transplantation: Ethical considerations}

\author{
Jennifer Flynn PhD ${ }^{1}$, Randi Zlotnik Shaul JD LLM PhD ${ }^{2,3}$, Mark D Hanson MD ${ }^{4}$, \\ Gregory $\mathrm{H}$ Borschel $\mathrm{MD}^{5,6}$, Ronald Zuker $\mathrm{MD}^{5,6}$
}

\begin{abstract}
J Flynn, R Zlotnik Shaul, MD Hanson, GH Borschel, R Zuker. Pediatric facial transplantation: Ethical considerations. Plast Surg 2014;22(2):67-69.
\end{abstract}

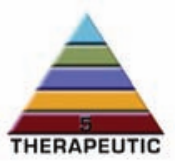

Facial transplantation is becoming increasingly accepted as a method of reconstructing otherwise unreconstructable adult faces. As this modality is made more available, we must turn our attention to pediatric patients who may benefit from facial transplantation. In the current article, the authors present and briefly examine the most pressing ethical challenges posed by the possibility of performing facial transplantation on pediatric patients. Furthermore, they issue a call for a policy statement on pediatric facial transplantation. The present article may serve as a first step in that direction, highlighting ethical issues that would need to be considered in the creation of such a statement.

\section{La greffe de visage en pédiatrie : des considérations éthiques}

La greffe de visage est une méthode de plus en plus acceptée pour reconstruire des visages adultes autrement impossibles à reconstruire. Puisque cette modalité devient plus accessible, nous devons nous pencher sur les patients d'âge pédiatrique qui pourraient en profiter. Dans le présent article, les auteurs présentent et analysent brièvement les problèmes éthiques les plus pressants liés à la possibilité d'une greffe de visage chez des patients d'âge pédiatrique. Ils réclament également la rédaction d'un document de principes sur le sujet. Le présent article peut constituer une première étape dans cette direction, car il souligne les enjeux éthiques qu'il faudrait évaluer lors de la préparation d'un tel document.

Key Words: Ethics; Facial transplantation; Pediatrics

\begin{abstract}
A $\mathrm{s}$ facial transplantation is becoming increasingly accepted as a method of reconstructing otherwise unreconstructable adult faces, we must turn our attention to its use in children $(1,2)$. In the present article, we analyze the most pressing ethical issues in pediatric facial transplantation. Furthermore, we issue a call for a policy statement on pediatric facial transplantation. Several programs have successfully transplanted faces in adults. More than 25 face transplants have been performed in adults, some of which are anatomically highly complex, including bone of both the upper and lower jaws, muscle, nerve, tongue and skin; the youngest recipient noted in the literature is a 19 year-old man who received a total facial transplant in Turkey in 2012. Many of the technical requirements for pediatric facial transplantation would be identical to adult cases; therefore, pediatric face transplantation is now technically feasible. However, the ethics of pediatric facial transplantation must be carefully considered before offering this treatment to the family of a child and to the child himself/ herself. In the current article, we highlight the ethical issues that would need to be considered before offering this treatment.

Given the risks and uncertainty associated with facial transplantation, it should be considered only when other reconstructive procedures have failed to achieve an acceptable result or when it is determined by an expert team that reconstructive procedures will not achieve an acceptable result. Facial transplantation is indicated for patients for whom conventional, non-allotransplant-based techniques cannot sufficiently restore form and function (eg, patients with large facial burns, cases in which the complex central facial features are absent and patients with massive injury to the central face that would require dozens of operations to reconstruct).
\end{abstract}

\section{THE RISK VERSUS BENEFIT ANALYSIS}

The weighing of risks and benefits is particularly complex within this context. At least two of the 25 patients who underwent facial transplantation died of complications related to their transplant. One complicating factor both within the general and pediatric populations is the fact that the procedure is not necessary to preserve life. While not strictly life saving, it may be considered so life enhancing that considerable risk may be taken in the hopes of achieving life-altering benefit (1). For example, one group concluded that facial transplantation could add 20 quality-adjusted life years compared with having a significant facial deformity (3). Furthermore, it is important to recognize functional gains: patients with central facial defects have difficulty breathing, speaking, chewing and swallowing, and often have an impaired sense of smell. The deformities, if untreated or undertreated, will significantly hinder a patient's quality of life.

Facial transplantation is similar to hand transplantation in that neither are life-saving interventions but offer rather the chance to improve lifestyle and function. There is currently a substantive body of literature on hand transplantation, albeit few studies on pediatric hand transplantation. Just as hand transplants have improved quality of life in adult recipients, it is also quite possible that pediatric hand transplants will provide similar improvements in quality of life. Similarly, the impressive gains in quality of life following facial transplantation in adults may also be replicated in children. As more face transplants are performed in children, the literature surrounding them, similar to adult hand and face transplants, would naturally continue to grow. Again, only highly selected pediatric patients and families would be chosen for facial transplantation. They would need to possess the resilience and resourcefulness needed to endure the surgery, postoperative care and the chronic immunosuppression, along with its attendant risks of comorbidity, to succeed.

In Canada, for example, taxpayers will ultimately fund these costly procedures and will also fund the lifelong immunosuppression and the management of downstream problems arising from such transplants. From a societal perspective, it may not be ethical to offer such expensive procedures, especially when their benefit is unproven. Nevertheless, in certain highly selected patients for whom no alternative exists, face transplantation may, in fact, be both cost effective and ethical. For example, many individuals with severe facial deformities may routinely undergo dozens of very large reconstructions, yet still experience major stigmata from their condition. Facial transplantation

${ }^{1}$ Division of Community Health and Humanities, Faculty of Medicine, Memorial University, St John's, Newfoundland and Labrador; ${ }^{2}$ Department of Bioethics, The Hospital for Sick Children; ${ }^{3}$ Department of Paediatrics; ${ }^{4}$ Department of Psychiatry, Faculty of Medicine, University of Toronto; ${ }^{5}$ Division of Plastic and Reconstructive Surgery, The Hospital for Sick Children; ${ }^{6}$ Department of Surgery, University of Toronto, Toronto, Ontario

Correspondence: Dr Jennifer Flynn, Division of Community Health and Humanities, Faculty of Medicine, Memorial University, 300 Prince Phillip

Drive, St John's, Newfoundland and Labrador A1B 3V6. Telephone 709-777-2043, fax 709-777-7382, e-mail jflynn@mun.ca 
offers a chance - in a single stage - to alleviate much of that suffering with a single, albeit large and risky, surgical procedure. Many adults have progressed to lead productive lives following facial transplant and, therefore, it stands to reason that children may also similarly benefit.

The analysis of the risks and benefits is further complicated within pediatrics by the following factors.

\section{Medical uncertainty}

The pediatric context increases the level of medical uncertainty involved in facial transplantation. First, there are fewer possible 'exit strategies' - strategies to use in the event of transplantation failure than when patients are adults. Compared with adults, children have large heads in proportion to their bodies. Any exit strategy that involves the transfer of muscles or other tissues from the rest of the body to the face will incur a challenge, with there being relatively more face to cover, and less body available for transfer compared with with adults. Additionally, the preferred tissues that one would use in such an event are comparatively less robust in small children (eg, the latissimus dorsi muscle flap could be difficult to use in a small child because it is so thin). Similarly, the omentum, the fatty tissue in the abdomen, can be used as a free flap to cover defects; in small children, it is often too thin and small relative to the area of the face. Nevertheless, in the event that a facial transplant should fail, alternative strategies, such as multiple emergent free tissue transfers or emergent listing for retransplant (as sometimes performed for solid organ transplants), are available and can be applied as needed.

In contrast to adult facial transplants, young children's facial transplants could still be growing at the time of transplant. In transplanting bone, which facial transplantation will sometimes involve, it is difficult to predict which bone will grow and which will not because it is difficult to predict whether growth centres are being transplanted in the growing facial skeleton. Three-dimensional computed tomography scanning combined with computer-aided design and rapid prototyping has become popular in craniofacial surgery and may assist greatly in determining which regions can be expected to grow over time (4).

\section{Longevity of the graft}

Transplanted faces may have limited lifespans. Accordingly, the performance of this procedure on a child could mandate subsequent retransplantation given the patient's young age. The effect on the risk-benefit balance of the possibility of additional (and possibly multiple) transplant(s) of the face is profound, given the psychosocial significance of the face in conjunction with the young age of the patients. A second facial transplant entails a child receiving a new face at a new age and, therefore, at a new psychosocial developmental stage compared with the first transplant. The attendant struggles with personal identity and social integration are not only imposed on a child for a second time but under new psychosocial conditions. This must be factored into the risk-benefit analysis for any particular patient. Similarly, there may be some question about the appropriateness of the use of immunosuppression for a (merely) life-enhancing (as opposed to life-saving) procedure. We recognize that the burden of lifelong immunosuppression is, in fact, an additional factor in the risk-benefit analysis. Despite recent advances in immunosuppression, it is a major burden at present, particularly for children, and it should certainly affect decision making. Nevertheless, novel investigational techniques, such as bone marrow and stem cell transplantation, hold promise in minimizing the side effects of immunosuppressive regimens; future patients may benefit greatly from such strategies.

\section{ISSUES SURROUNDING INFORMED CONSENT}

Informed consent is a notoriously difficult issue when children are involved. There is the question of when a child may be capable of acting autonomously, and accepting for himself/herself this high-risk intervention (given, among other issues, the serious risks posed by lifelong immunosuppression). Generally speaking, judgments about a child's ability to act autonomously in this regard will have to be made on a case-by-case basis. This is the case in Canadian jurisdictions where there is no age of consent. Even in Canadian jurisdictions where there is an age of consent, when it comes to older children and teenagers, case-by-case assessments ought to be made given the possibility of, for example, a patient being deemed a 'mature minor'. It seems quite clear to us that given the complexity of what is involved, it is unlikely that any preteen or younger candidate would be deemed capable of providing autonomous consent to the procedure.

We must also grapple with the following two considerations when considering consent.

\section{The possibility of future resentment}

In many or even most cases, a patient's parent will be consenting to the procedure. Future resentment on behalf of the patient toward the parent (or other decision maker) is a possibility. For example, the patient may resent that the choice resulted in him or her having to take certain medications for a lifetime, or the patient may resent having this 'new face'. There is, perhaps, equally as good a chance that the patient will respond with gratitude; however, we still must take seriously this chance of future resentment. We ought also to recognize the unique form this resentment could take given the connection between the face and personal identity (as discussed below). Involving even young children in their assessment for this treatment may be a way to prevent resentment, although the severity of the child's condition may render such involvement impossible.

\section{Donation and informed consent}

There is the further issue of informed consent vis-à-vis donation. The uncertainty of facial transplant outcomes is relevant to informed consent in that a donor - generally the parent or other adult decisionmaker - will look for assurance that the donation will be put to good use. It is, in our view, reasonable to assume that grieving parents will want assurance that their donation of their child's face is being put to good use, even if a guarantee is not expected. However, in the case of pediatric facial transplantation, such assurance would be more difficult to give compared with the adult situation, given the issues reviewed above. Grieving parents may be motivated to make such a donation only on the assurance that the donation would very likely result in a good outcome for the recipient. The likelihood of parents consenting to such a donation on behalf of their child may well correlate with the level of assurance parents are offered (or, at least, the level of assurance they perceive they have been offered). To the extent that a donor's being 'informed' relates to that donor's being given a sense of expected outcomes, informed consent within this context is problematic. This issue of uncertainty pertains to much of medical practice. However, given the novelty of pediatric facial transplantation, uncertainty is particularly pronounced. Furthermore, there is an emotional dimension to parents donating their recently deceased child's face that may well heighten the perceived need by donors for assurance about donation outcomes. Mental health personnel would need to work closely with the donor families to help them work through this issue.

\section{ISSUES SURROUNDING PERSONAL IDENTITY}

The face is closely connected to personal identity, partly for psychological and emotional reasons, and also because certain accounts of personal identity equate its continuity with bodily continuity $(5-7)$. Furthermore, on some views, the face is closely related to identity formation (8). We often identify people with their faces, and individuals themselves tend to see their own face as a reflection of 'who they are' $(1,9)$. The face's psychosocial significance, in conjunction with the developing personal identity of the child or adolescent, means that social integration post-transplant could be more difficult for children than for adults; that the face is an external organ is no small matter $(1,6)$. Key areas in which visible differences may impact a child's development include parent-child attachment and family relationships, self-perceptions and social networks (10). How will a child's new appearance influence his/her relationships? How should 
such considerations play into the risk/benefit analysis? Follow-up and support pertaining to these issues must be considered an essential part of the ongoing treatment plan.

\section{CANDIDATE SELECTION}

\section{The speed of childhood development}

The speed with which children and adolescents pass through developmental stages may present a challenge when selecting recipients for facial transplantation. A child or adolescent may appear to be a suitable candidate at the time of assessment, with developmental changes influencing that suitability by the time of transplant. Key to addressing the vicissitudes of childhood development will be the evaluation of the patient within the family context because pediatric post-traumatic injury adjustment is positively associated with supportive families $(1,11-14)$ and psychological adjustment $(13,15)$. An ongoing assessment of patient assent could mitigate some emotional and psychological variances and fluctuations.

\section{The relevance of pretrauma psychological health}

The contribution of the psychological health of a potential recipient to the decision regarding suitability of that patient for the procedure is complex. Perhaps children who are psychologically healthy and resilient before transplantation will recover from the transplant more readily and benefit from the transplant, leading to the assertion that psychologically healthy and resilient children are the best candidates for the transplant. One may argue that to determine suitability in such a way is problematic, in that those psychologically healthy and resilient pre-trauma are likely to be considered better candidates, with those less psychologically healthy and resilient pretrauma considered worse candidates (6). Yet, such considerations are pertinent to pediatric facial transplantation in that the psychosocial challenges postfacial transplant are likely more profound compared with other transplants, heightening the relevance of a patient's pre- and posttransplant psychological state; also, as discussed above, the young age of the pediatric population suggests particular post-transplantation adjustment challenges. We suggest that children with severe facial disfigurement must exhibit sufficient psychological health and resilience to participate within the treatment plan. A specialized team of pediatric mental health professionals is necessary to make this determination because it will require assessment of the psychological impact of the child's severe facial disfigurement alongside the child's psychological health, resilience and capability to participate within their treatment plan.

\section{Reasons for donation}

What would drive a family to choose to donate their child's face? A parent may, for example, donate her child's face in the hopes of 'seeing my child's face again' (6). Furthermore, we ask what the transplant team should do in the event that the team deems the justification for donation (morally) dubious. Should a donation be refused on the grounds that the justifications are regarded to be problematic? Is the scarcity of the resource morally relevant? The scarcity issue should not be underestimated: the donors and recipients will need to be matched closely for age because of tissue growth rates, and also for complexion and sex. We expect that few parents or substitute decision makers will be willing to make this donation in the first place. At the same time, no patient, particularly no young patient whose substitute decision maker is in control of decision making, should be pressured into accepting a transplant, on the grounds, for example, that a perfect match has been found. Depending on the arrangements and protocols of specific transplantation programs, transplant teams may or may not be able to evaluate reasons why one wants to donate. When such evaluation does occur, however, it will be ethically complex. We suggest that the families should be asked why they wish to donate, and if concerns arise, that the transplant team discuss the matter with the coordinator of the organ procurement organization.

\section{PEDIATRIC FACIAL TRANSPLANTATION AND THE RESEARCH CONTEXT}

Facial transplantation should be undertaken in institutions with either institutional review boards (or their equivalent) or innovative procedural protocols that are equipped to review, approve and provide oversight. While the technical components of a face transplant are essentially standard practice for transplant and plastic surgeons, the procedure has not yet been performed on a child; therefore, research in pediatric facial transplantation should be conducted with institutional approval through an institutional review board.

On a related note, pediatric facial transplantation should be considered experimental. From an ethical point of view, future pediatric facial transplantation protocols should be accompanied with concomitant measurement of the outcomes that should include both physiological measures and patient-reported outcomes (ie, health-related quality of life).

\section{CONCLUSION}

Technological readiness is insufficient for the implementation of facial transplantation within pediatrics. The complex ethical issues associated with such transplants must be addressed. One way of achieving this would be through the development of a policy statement on this topic; the above analysis could inform such a statement.

INSTITUTION: This work originated at The Hospital for Sick Children and the University of Toronto, Toronto, Ontario.

\section{REFERENCES}

1. Gordon CR, Siemionow M, Papay F, et al. The world's experience with facial transplantation: What have we learned thus far? Ann Plast Surg 2009;63:572-8.

2. Murphy BD, Zuker R, Borschel G. Vascularized composite allotransplantation: An update on medical and surgical progress and remaining challenges. J Plast Reconstr Aesthet Surg 2013;66:1449-55.

3. Cugno S, Sprague S, Duku E, et al. Composite tissue allotransplantation of the face: Decision analysis model Can J Plast Surg 2007;15:145-52.

4. Ciocca L, Mazoni S, Fantini M, et al. CAD/CAM guided secondary mandibular reconstruction of a discontinuity defect after ablative cancer surgery. J Craniomaxillofac Surg 2012;40:e511-5.

5. Swindell JS. Facial allograft transplantation, personal identity and subjectivity. J Med Ethics 2007;33:449-53.

6. Huxtable R, Woodley J. Gaining face or losing face? Framing the debate on face transplants. Bioethics 2005;19:505-22.

7. Caplan A, Katz D. In brief: About face. Hastings Cent Rep 2003;33:8.

8. Baylis F. A face is not just like a hand: pace Barker. Am J Bioeth 2004:4:30-1.

9. Siemionow M, Sonmez E. Face as an organ. Ann Plast Surg 2008;61:345-52.

10. Rumsey N, Harcourt D. Visible differences amongst children and adolescents: Issues and interventions. Dev Neurorehabil 2007;10:113-23

11. Sheridan RL, Hinson MI, Liang MH, et al. Long-term outcome of children surviving massive burns. J Am Med Assoc 2000;283:69-73.

12. Landolt MA, Grubenmann S, Meuli M. Family impact greatest: Predictors of quality of life and psychological adjustment in pediatric burn survivors. J Trauma 2002;53:1146-51.

13. Noronha DO, Faust J. Identifying the variables impacting post-burn psychological adjustment: A meta-analysis. J Pediatr Psychol 2007;32:380-91.

14. Aitken ME, Tilford JM, Barrett KW, et al. Health status of children after admission for injury. Pediatrics 2002;110:337-42.

15. Vollrath M, Landolt MA. Personality predicts quality of life in pediatric patients with unintentional injuries: A 1-year follow-up study. J Pediatr Psychol 2005;30:481-91. 